\title{
Impacto del Uso de Audífonos para Adultos Mayores en Chile
}

\author{
Miguel A. Bustamante(1), Cristian L. Vidal(2) y Leopoldo P. López ${ }^{(3)}$ \\ (1) Escuela de Ingeniería Comercial, Facultad de Economía y Negocios, Universidad de Talca Campus \\ Lircay, Avenida Lircay S/N, Talca-Chile (e-mail: mabu@utalca.cl) \\ (2) Departamento de Computación e Informática, Facultad de Ingeniería, Universidad de Playa Ancha, \\ Avenida Leopoldo Carvallo 270, Playa Ancha, Valparaíso-Chile (e-mail: cristian.vidal@upla.cl) \\ (3) Instituto de Investigación y Desarrollo Educacional, IIDE, Universidad de Talca Campus Lircay, Avenida \\ Lircay S/N, Talca-Chile (e-mail: Ilopez@utalca.cl)
}

Recibido Dic. 5, 2013; Aceptado Feb. 6, 2014; Versión final recibida Feb. 11, 2014

\begin{abstract}
Resumen
Este trabajo estudia la satisfacción usuaria de una muestra representativa de adultos mayores urbanos que reciben audífonos por la vía del programa Garantías Explícitas en Salud (GES). Se utilizó el constructo operacional descrito en la guía clínica del Ministerio de Salud de Chile, MINSAL № 56 del año 2007, llamada "Hipoacusia bilateral en personas de 65 años y más que requieren audífonos", y que define los ítems a evaluar. Se aplicó el "Cuestionario Internacional de Resultado de Uso de Audífonos CIRUA". Se consideró como muestra aquellos individuos que tienen más de un año utilizando el dispositivo desde su entrega. Finalmente, los adultos mayores valoran el haber recibido sus respectivos audífonos, reconocen su utilidad y aprecian cómo les ha cambiado la vida y sus rutinas habituales: También han reducido caídas y manifiestan tener ahora una mejor calidad de vida.
\end{abstract}

Palabras clave: adulto mayor, audífonos, hipoacusia, GES, guía clínica

\section{Impact of Using Earphones for Older People in Chile}

\begin{abstract}
This paper studies the user satisfaction of a representative sample of urban elderly who receive hearing aids via the GES program (Explicit Health Guarantees). We used the operational construct of the Health Ministry of Chile, MINSAL clinical guideline No. 56 of 2007, named "Bilateral hearing loss in people of 65 years and older who require earphones", and that defines the items to be evaluated. The so-called "International Survey of Use of Earphones CIRUA" was applied. The sample considered in the study was defined as elderly people who have used the device for more than one year since its delivery. Finally, elderly people value the fact of receiving their hearing aids, recognize its usefulness, and appreciate how it has changed their life and their normal routines. They also indicate that falls have reduced falls and indicate that they have at present a better quality of life.
\end{abstract}

Keywords: urban elderly, earphones, hearing loss, GES, clinical guideline 


\section{INTRODUCCIÓN}

En Chile el $75 \%$ de los adultos mayores presenta algún nivel de pérdida auditiva. En los mayores de 80 años, la prevalencia fue del 90\% además, según la Sociedad Chilena de Otorrinología, 9 de cada 10 casos de este déficit es de tipo sensorio-neural (Cañete, 2006). Se estima que el $80 \%$ de las personas mayores de 65 años experimentan al menos un problema crónico y, que en ciertas condiciones otológicas, tienen mayor prevalencia en los adultos mayores si se compara con jóvenes (Puente, 1994) dando pié a la presbi-acusia (Cañete, 2006) que como resultado final conduce al aislamiento (Tamblay et al., 2008), siendo éste un problema que es susceptible de rehabilitación mediante auxiliares auditivos eléctricos (Contreras, 2006).

La discusión pública de este tema constituye un importante punto de partida para generar nuevos aprendizajes (Salas, 2013) del concepto de salud descrito en términos de un estado de bienestar físico, mental y socio-económico, cuya carencia pueda afectar la calidad de vida y/o la recuperación de ésta (Gómez Pérez, 2006). En este escenario, la intervención temprana mediante el uso de audífonos (S.E.G-G Geriatría XXI, 2000) se consideran una herramienta eficaz en la promoción de un envejecimiento con calidad de vida (Ferré et al., 2002). Según el Censo año 2002 en Chile, a nivel de la provincia de Talca se calcula un 10,85\% de adultos mayores (Censo Chile, 2002), y según la misma fuente, a nivel comunal, Talca alcanza un $8,2 \%$ de incapacidad auditiva dentro del grupo etáreo adulto mayor. Según información de Auditrón, empresa encargada de hacer efectiva la entrega y seguimiento de los audífonos a los adultos mayores registrados, estos datos se confirman al haberse entregado 1637 unidades de audífonos superando la meta sanitaria del año 2008 (Auditrón, 2013). Es importante señalar además que, según estudios Fondecyt (Fondecyt 1080146; Fondecyt 1100611), se confirma la relevancia de "las condiciones de vida" que definen "componentes objetivos" y la "satisfacción personal con las condiciones de vida" que definen "componentes subjetivos" de lo que las personas aprecian conforme su propia escala de valores y aspiraciones (Castellón y Sánchez del Pino, 2001), para dar forma a un indicador de calidad que forma parte de un modelo de salud familiar (Oliva e Hidalgo, 2004).

Sobre la base de lo señalado, el objetivo principal de la presente investigación es estudiar la satisfacción usuaria de una muestra de la población de adultos mayores urbanos registrados en FONASA de la Comuna de Talca que reciben audífonos por la vía del programa GES. Sin considerar diferencias geográficas y su impacto en la población objetivo, se afirma que estos resultados pueden ser extrapolados a la población de adultos mayores en Chile. Este trabajo se organiza de la siguiente forma. La siguiente sección entrega detalles acerca del objetivo de este trabajo y de la metodología usada para su alcance. Luego, una sección que detalla elementos utilizados para el análisis de datos de la muestra, esto es, estadística descriptica y estadística inferencial. Posteriormente, una sección con los resultados obtenidos en este estudio considerando elementos demográficos y detalles de la encuesta aplicada junto con sus resultados. Finalmente, una sección que presenta las principales conclusiones.

\section{OBJETIVOS Y METODOLOGÍA}

La presente investigación estudia la percepción del Bienestar Subjetivo (BS) (Diener et al., 1999; Diener y Lucas, 2000; Diener, 1984) de la satisfacción personal de una muestra de los adultos mayores por el uso de audífonos. La muestra de la población objetivo corresponde a adultos mayores de la ciudad de Talca, Chile, quienes han accedido a audífonos por beneficio GES. Este trabajo analiza los componentes "desempeño auditivo subjetivo y satisfacción en adultos mayores" (Diener, 1984; Diener et al., 1985) con el fin de, dado el estudio muestral, describir algunas de las condiciones de vida de este grupo etáreo, haciendo coincidir componentes subjetivos y objetivos en esta dimensión particular de la calidad de vida (Castellón y Sánchez del Pino, 2001). Cabe señalar que estos resultados, dadas las diferentes condiciones demográficas, sociales, y culturales de Chile, no son completamente extrapolables para todo Chile.

Esta investigación revisa los principales cambios en la calidad de vida de los adultos mayores que han accedido a la entrega de audífono por beneficio GES, particularmente, en la comuna de Talca entre los años 2008 - 2011 con la idea de extrapolar dichos resultados a otras ciudades de Chile con una población de adultos mayores con características similares. Para ello fue preciso, caracterizar socio-económicamente la población que accede al beneficio, identificar enfermedades crónicas o neurológicas de la población que accede al beneficio y determinar cuál fue el factor desencadenante para acceder a la entrega del audífono. Así mismo, determinar si la entrega de audífono ha implicado cambios en su calidad de vida e indicar cuál es la causa de no utilización de audífono, para finalmente, establecer propuestas en beneficio en la calidad de vida de los adultos mayores.

Se hizo una revisión exhaustiva de la guía clínica MINSAL Nº 56 del año 2007 (Minsal, 2013); correspondiente a la patología "Hipoacusia bilateral en personas de 65 años y más que requieren audífonos" para definir ítems a evaluar en la encuesta aplicada a la muestra. 
Como fuentes de información secundaria, se recolectó datos desde la oficina GES del Hospital Regional de Talca y de una organización externa adjudicataria para la entrega de audífonos en este periodo (Auditrón, 2013), la que reportó un número de 50 datos de adultos mayores sujeto de la entrega y se obtuvo el registro de números telefónicos y direcciones de quienes se encuentran formalmente incorporados al sistema oficial de entrega de Audífonos GES por parte de esta organización encomendada por FONASA.

Se planificaron entrevistas directas con el instrumento prediseñado considerando como universo a los adultos mayores que han recibido el beneficio GES de entrega de audífonos con prestador FONASA y se consideró como muestra aquellos que tienen más de un año desde la entrega del beneficio periodo comprendido desde agosto del 2008 y hasta el año 2011. Se realizó el diseño de un cuestionario combinado de recogida de datos, conformado por el "Cuestionario Internacional de Resultado de Uso de Audífonos CIRUA" (Cox et al., 2002) que incluye una escala Likert de 5 puntos crecientes (Likert, 1932). El instrumento diseñado fue sometido a diversas validaciones a través de consultas a expertos y piloteo mediante su aplicación a un grupo control a fin de corregir eventuales errores de diseño y asegurar su comprensión. Finalmente fue aplicado, previa validación y correcciones de acuerdo con las observaciones recogidas.

\section{ANÁLISIS DE DATOS}

Se procedió al análisis socio-demográfico de la muestra sobre la base de los datos recogidos en las entidades informantes, Hospital Regional de Talca y Organización adjudicataria para la distribución de los Audífonos a los Adultos mayores debidamente registrados.

\section{Estadística Descriptiva}

Para esta síntesis estadística, se consideró la verificación de los registros válidos y perdidos, así como las cifras de población urbana, rural y total, respecto de las cuales se determinó las cifras del sistema, junto con las respectivas frecuencias y porcentajes válidos y acumulados, para finalmente determinar los estadísticos descriptivos media, mediana y moda.

\section{Estadística Inferencial}

Como se presenta en los resultados de la investigación, se realizó un "Análisis de Factores de Componentes Principales" de los datos (Ferrando, 1994; García et al., 2000), por cuanto dicho método permite el análisis de los datos agrupándolos en factores constituidos por componentes principales validados por cargas estadísticas representativas.

La aplicación del "Análisis de Factores de Componentes Principales" fue posible ya que es compatible en esencia con la Escala de Bienestar Psicológico (Diener et al., 1985) que trabaja con 13 ítems y con tres opciones de respuesta, y la Escala de Bienestar Subjetivo (Diener et al., 1999), que se aplica a través de una escala tipo Likert (Likert, 1932), en este caso con cinco opciones de respuesta. Además, C. Ryff presenta en 'Happiness is Everything, or Is It?' (1989) y en 'In the Eye of the Beholder: Views of Psychological well-Being in Middle and old-Aged Adults' (1989) una escala de bienestar psicológico EBP que utiliza una secuencia de 1 (completamente en desacuerdo) a 6 (completamente de acuerdo). Por otra parte, la OHQ (Oxford Happyness Questionaire) (Diener y Lucas, 2000) también aplica una escala de 1 a 6. Finalmente, la Escala de Felicidad Subjetiva, EFS, contempla, así mismo, una escala Likert de 1 a 7 puntos (Likert, 1932). Es decir, definir una escala de factores de componentes principales era completamente viable.

A este análisis se agregó la estandarización de los resultados obtenidos en algunas de las aplicaciones metodológicas a fin de comparar los resultados y determinar las distancias entre los elementos analizados.

\section{RESULTADOS DE LA INVESTIGACIÓN}

Dentro del periodo de validación y aplicación del instrumento pasan dos años en el cual se produce el terremoto del 27/F y que al momento de proceder a las aplicaciones del cuestionario, produjo dificultades para encontrar las direcciones originales y, por ende, encuestar al total de los usuarios de los que se disponía información. Como resultado, no se logra pesquisar más usuarios ya que ahora el proceso está en manos de una nueva empresa encargada por licitación que se hace cargo de la entrega de los audífonos en el territorio de la Comuna de Talca. A continuación, se presenta un análisis de factores de componentes principales del CIRUA, un análisis demográfico de la muestra, un análisis relativo a enfermedades y nivel educacional de la muestra, para finalmente presentar un análisis estadístico del Cuestionario Internacional de Resultado de Uso de Audífonos (CIRUA). 


\section{Análisis de Factores de Componentes Principales del Cuestionario Internacional (CIRUA)}

Con el propósito de analizar en profundidad, tanto las razones como los beneficios que reporta a los adultos mayores el uso de audífonos, se procedió a la determinación de los factores de "Componentes Principales del Cuestionario Internacional de Resultado de Uso de Audífonos" CIRUA. La tabla 1 detalla los resultados del análisis estadístico de análisis de factores de componentes rotados. Se detalla el análisis de factores del CIRUA y de la matriz de componentes rotados. Se determinaron 3 factores que sintetizan y focalizan las respuestas recogidas de parte de los adultos mayores.

Tabla 1: Factores de componentes principales del cuestionario CIRUA

\begin{tabular}{|c|c|c|}
\hline & \multicolumn{2}{|l|}{ Factor 1 "Escucha satisfactoria en la vida rutinaria" } \\
\hline$N^{\circ}$ & Componentes (CIRUA) & Carga \\
\hline 3 & $\begin{array}{l}\text { Piensa una vez más en la situación en la usted realmente desea escuchar mejor. } \\
\text { Cuando utiliza el (los) audífono(s) actual(es) ¿Cuánta dificultad tiene todavía en } \\
\text { esa situación? }\end{array}$ & 0,732 \\
\hline \multirow[t]{4}{*}{5} & $\begin{array}{l}\text { En las últimas dos semanas con el (los) audífono(s) que utiliza actualmente, } \\
\text { ¿Cuánto ha afectado su rutina diaria su dificultad auditiva? }\end{array}$ & 0,856 \\
\hline & Varianza Total Explicada (Fi) & 25,515 \\
\hline & Varianza Acumulada $\sum(\mathrm{Fi})$ & 25,515 \\
\hline & \multicolumn{2}{|l|}{ Factor 2 "Escuchar para disfrutar de la vida" } \\
\hline$N^{\circ}$ & Componentes (CIRUA) & Carga \\
\hline 2 & $\begin{array}{l}\text { Piense en una situación donde usted Realmente hubiera querido escuchar mejor } \\
\text { antes de obtener su(s) audífono(s) actual(es). En las últimas dos semanas } \\
\text { ¿Cuánto le ha ayudado el (los) audífono(s) en esa situación? }\end{array}$ & 0,847 \\
\hline \multirow[t]{4}{*}{7} & $\begin{array}{l}\text { Considerándolo todo, ¿Cuánto ha cambiado su manera de disfrutar la vida el (los) } \\
\text { audífono(s) que utiliza actualmente? }\end{array}$ & 0,768 \\
\hline & Varianza Total Explicada (Fi) & 22,653 \\
\hline & Varianza Acumulada $\sum(\mathrm{Fi})$ & 48,168 \\
\hline & \multicolumn{2}{|l|}{ Factor 3 "A pesar de todo escuchar mejor vale la pena" } \\
\hline$N^{\circ}$ & Componentes (CIRUA) & Carga \\
\hline 1 & $\begin{array}{l}\text { Piense cuanto ha utilizado usted su(s) audífono(s) actual(s) en las últimas dos } \\
\text { semanas. En un día común ¿Cuántas horas ha usado usted el (los) audífonos? }\end{array}$ & 0,836 \\
\hline 4 & $\begin{array}{l}\text { Considerándolo todo piensa, ¿Piensa usted que su(s) audífono(s) actuales vale(n) } \\
\text { la pena? }\end{array}$ & 0,423 \\
\hline \multirow[t]{3}{*}{6} & $\begin{array}{l}\text { En las dos semanas pasadas con su(s) audífono(s) actual(es), ¿Cuánto piensa } \\
\text { usted que su dificultad auditiva ha molestado a otras personas? }\end{array}$ & $-0,705$ \\
\hline & Varianza Total Explicada (Fi) & 18,400 \\
\hline & Varianza Acumulada $\sum(\mathrm{Fi})$ & 66,569 \\
\hline
\end{tabular}

Además, la tabla 1 presenta 3 factores determinados con títulos descriptivos que los identifican y que en conjunto explican el $66,6 \%$ de la varianza total. Además, estos factores señalan, para cada caso, los respectivos componentes principales con las cargas representativas del grado de correlación de dichos componentes con los factores que ellos definen. Gracias a este análisis de factores, se permite analizar en profundidad, tanto las razones como los beneficios que reporta a los adultos mayores el uso de audífonos.

\section{Análisis Socio-Demográfico de la Muestra}

Como muestra la tabla 2, existe una mayoría de usuarios que pertenecen a la comuna de Talca con domicilio en la zona urbana $(84,4 \%)$, con predominio de sexo femenino $(68,8 \%)$ y que vive con algún familiar $(90,6 \%)$. Una breve contrastación confirma el hecho que la relación a población urbana versus rural, se ajusta porcentualmente al estándar de población correspondiente a la comuna de Talca. La tabla 2 indica que la media del ingreso se ubica en la escala entre $100-200$ mil pesos y son dependientes en un $59,4 \%$. En su mayoría se ubican con edades en una escala entre 75 - 85 años $(43,8 \%)$ siendo el grupo de mayores de 85 años de tan sólo un 15,6\%. Además, un destacado $71,9 \%$ viven en una vivienda propia, el $46,9 \%$ son viudas(os), un 31,3 permanece casado/a. 
Tabla 2: Demografía de la muestra ( Depen.= Dependiente)

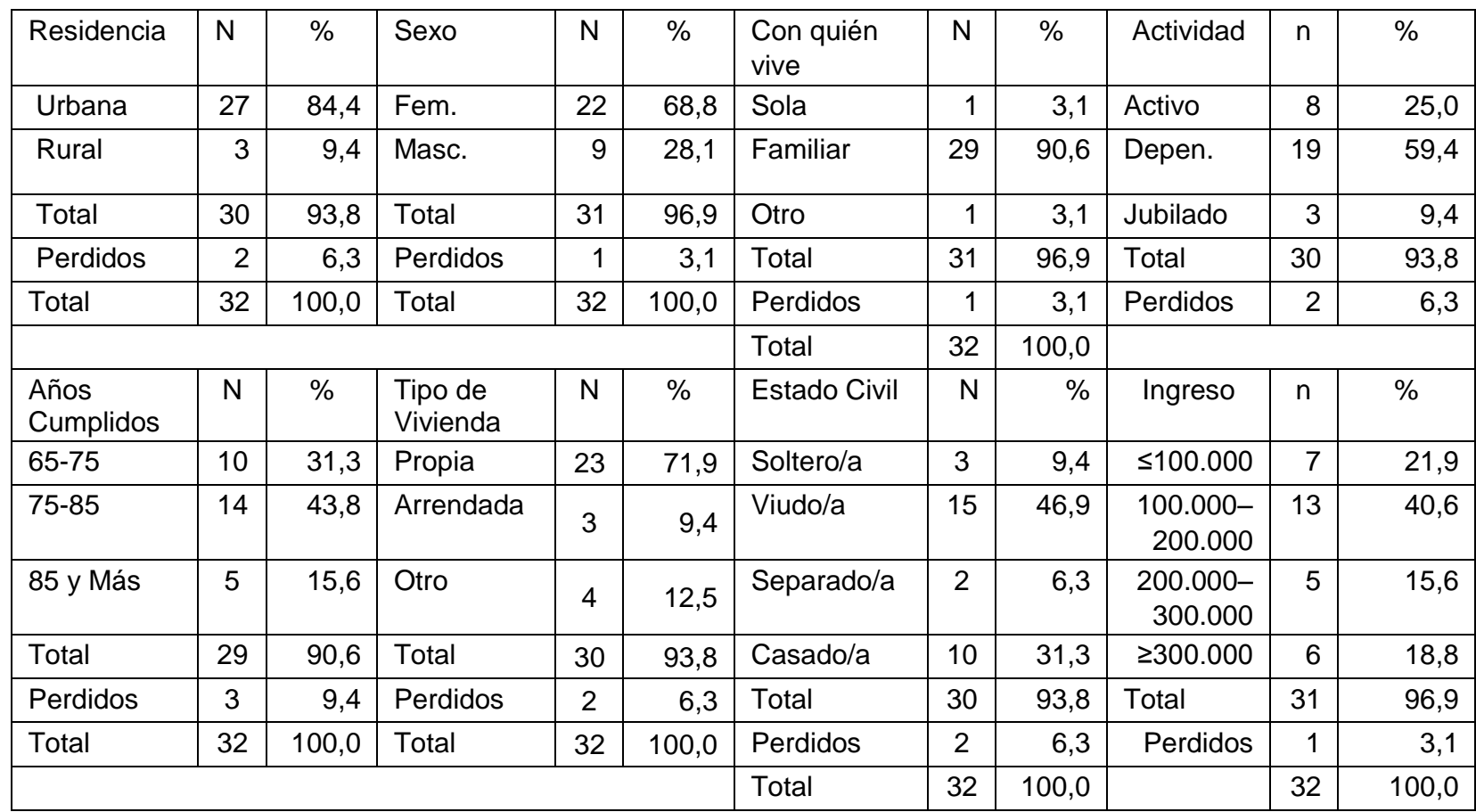

Finalmente, como muestra la tabla 3 , en su mayoría, $21,9 \%$ señalan un nivel educacional básico. Sin embargo, en esta pregunta, el total de respuestas no contestadas del ítem Perdidos llega a 59,4\%, lo que puede significar simplemente, no haber respondido la pregunta hasta reflejar un cierto grado de analfabetismo.

Tabla 3: Enfermedades de Base y Nivel Educacional.

\begin{tabular}{|l|r|r|l|r|r|l|r|r|r|c|c|}
\hline $\begin{array}{l}\text { Enfermedad } \\
\text { Crónica }\end{array}$ & $\mathrm{n}$ & \multicolumn{1}{|c|}{$\%$} & $\begin{array}{l}\text { Enfermedad } \\
\text { Neurológica }\end{array}$ & $\mathrm{N}$ & $\%$ & $\begin{array}{l}\text { Alteración } \\
\text { Visual }\end{array}$ & $\mathrm{n}$ & $\%$ & $\begin{array}{l}\text { Nivel } \\
\text { Educacional }\end{array}$ & $\mathrm{n}$ & $\%$ \\
\hline Sí & 21 & 65,6 & Parkinson & 3 & 9,4 & SI & 28 & 87,5 & Básico & 7 & 21,9 \\
\hline No & 7 & 21,9 & Demencia Senil & 1 & 3,1 & NO & 2 & 6,3 & Medio & 4 & 12,5 \\
\hline Otra & 1 & 3,1 & Otras & 1 & 3,1 & Total & 30 & 93,8 & Superior & 2 & 6,3 \\
\hline Total & 29 & 90,6 & Total & 5 & 15,6 & Perdidos & 2 & 6,3 & Total & 13 & 40,6 \\
\hline Perdidos & 3 & 9,4 & Perdidos & 27 & 84,4 & Total & 32 & 100,0 & Perdidos & 19 & 59,4 \\
\hline Total & 32 & 100,0 & Total & 32 & 100,0 & & & & Total & 32 & 100,0 \\
\hline
\end{tabular}

Se aprecia además en la tabla 3 que en cuanto a las enfermedades crónicas, un $87,5 \%$ presenta alteración visual, un $65,6 \%$ padece alguna de las enfermedades neurológicas de las cuales, la más frecuente es el Parkinson con un $9,4 \%$. Es importante mencionar el gran porcentaje de problemas visuales (87,5\%) asociados a los problemas de presbiacusia lo que puede deteriorar aún más la calidad de vida del adulto mayor y puede influir en forma negativa al momento de querer utilizar su audífono. Además, aunque en su mayoría el nivel educacional es básico, este nivel es directamente proporcional a los encontrados en los estudios de población de la región del Maule, esto es importante de considerar cuando se quiere entregar información sobre una prótesis cuyo aparato requiere de una determinada secuencia de instrucciones para su uso cuya dificultad de compresión técnica reduce o desalienta a los adultos mayores para lograr su mejor uso.

\section{Análisis del Resultado de Uso de Audífonos de los Adultos Mayores}

En General, como se muestra en la tabla 4, se observa un aumento en la satisfacción por el uso de audífonos según lo señalado por los Adultos mayores, sin embargo, se observan distinciones en la forma como éstos evalúan el uso de sus audífonos. La tabla 4 muestra que cinco preguntas del cuestionario (1, 2, 4,6 y 7) alcanzaron las más altas frecuencias relativas concentrando el valor de la media en el nivel 5 y dos preguntas ( 3 y 5 ) alcanzaron percepciones medias ubicadas en la escala 3 de la escala Likert utilizada (Likert, 1932). 
Tabla 4: Análisis Estadístico del Cuestionario Internacional de Resultado de Uso de Audífonos (CIRUA). Nota: Escala Likert (Likert, 1932), de 5 Puntos - Siglas: Val.: Válidos / Per.: Perdidos / Med.: Media / Mdn.: Mediana / Mod.: Moda

\begin{tabular}{|c|c|c|c|c|c|c|c|c|c|c|c|}
\hline $\mathrm{N}^{\circ}$ & Concepto & 1 & 2 & 3 & 4 & 5 & Val. & Per & Med & Mdn & Mod \\
\hline 1 & $\begin{array}{l}\text { Piense cuanto ha utilizado Ud. } \\
\text { su(s) audífono(s) actual(es) en las } \\
\text { últimas dos semanas. En un día } \\
\text { común ¿Cuantas horas ha usado } \\
\text { sus audífonos? }\end{array}$ & $3,2 \%$ & $6,5 \%$ & - & $9,7 \%$ & $80,6 \%$ & 31 & 1 & 4,58 & 5 & 5 \\
\hline 2 & $\begin{array}{l}\text { Piense en una situación donde } \\
\text { usted Realmente hubiera querido } \\
\text { escuchar mejor antes de obtener } \\
\text { sus actuales audífonos. En las } \\
\text { últimas dos semanas, ¿Cuánto } \\
\text { habrían ayudado los audífonos en } \\
\text { esa situación }\end{array}$ & - & - & $19,4 \%$ & $22,6 \%$ & $58,1 \%$ & 31 & 1 & 4,39 & 5 & 5 \\
\hline 3 & $\begin{array}{l}\text { Piensa una vez más en la situación } \\
\text { anterior. Cuando utiliza sus } \\
\text { audífonos actuales ¿Cuánta } \\
\text { dificultad tiene aún en esa } \\
\text { situación? }\end{array}$ & $9,7 \%$ & $6,5 \%$ & $35,5 \%$ & 22,6 & $25,8 \%$ & 31 & 1 & 3,48 & 3 & 3 \\
\hline 4 & $\begin{array}{l}\text { Considerándolo todo, ¿Piensa Ud. } \\
\text { que sus audífonos actuales valen la } \\
\text { pena? }\end{array}$ & - & - & $3,2 \%$ & $19,4 \%$ & $77,4 \%$ & 31 & 1 & 4,74 & 5 & 5 \\
\hline 5 & $\begin{array}{l}\text { En las últimas dos semanas con los } \\
\text { audífonos que utiliza actualmente, } \\
\text { ¿Cuánto ha afectado su rutina } \\
\text { diaria su dificultad auditiva? }\end{array}$ & $16,1 \%$ & $12,9 \%$ & $32,3 \%$ & $19,4 \%$ & $19,4 \%$ & 31 & 1 & 3,13 & 3 & 3 \\
\hline 6 & $\begin{array}{l}\text { En las dos semanas pasadas con } \\
\text { sus audífonos actuales, ¿Cuánto } \\
\text { piensa Ud. que su dificultad } \\
\text { auditiva ha molestado a otras } \\
\text { personas? }\end{array}$ & - & $16,7 \%$ & $26,7 \%$ & $10,0 \%$ & $46,7 \%$ & 32 & 2 & 3,87 & 4 & 5 \\
\hline 7 & $\begin{array}{l}\text { Considerándolo todo, ¿Cuánto ha } \\
\text { cambiado su manera de disfrutar la } \\
\text { vida el uso de sus audífonos? }\end{array}$ & - & $3,2 \%$ & $6,5 \%$ & $35,5 \%$ & $54,8 \%$ & 31 & 1 & 4,42 & 5 & 5 \\
\hline
\end{tabular}

Alcanzando la escala 5 de la escala Likert (Likert, 1932) con una media de 4,5; un 80,6\% de ellos señalan haber utilizado sus respectivos audífono en el transcurso de las últimas dos semanas, incluso habiéndolos utilizados una cuantas horas claramente identificables para ellos. Al mismo tiempo y a igual nivel de la escala, con un 4,3 de media, indican con un $58,1 \%$ de frecuencia relativa que les ha permitido escuchar mejor sintiendo que su uso les ha ayudado en diversas situaciones. En esta misma tendencia perceptiva y llegando a una media de 4,7 puntos de la escala Likert (Likert, 1932), un 77,4\% de los entrevistados considera que, por encima de todo, el uso de sus audífonos vale la pena y el esfuerzo realizado. Del mismo modo, pero ahora con una media de 3,86; un $46,7 \%$ indica que en las dos semanas pasadas sus audífonos les han significado una cierta dificultad auditiva y al mismo tiempo creen que su uso ha molestado a otras personas. Sin embargo, alcanzando el valor superior de la escala, esto es, escala 5, y con una media de 4,4 ; un destacable $54,8 \%$ considera que, por sobre todo, el uso de sus audífonos ha cambiado positivamente su manera de disfrutar la vida.

Consecuente con lo señalado, un $35,5 \%$ de los adultos mayores piensa una vez más que, aunque ellos realmente desean escuchar mejor, en las oportunidades en que utilizan sus audífonos todavía enfrentan ciertas dificultades lo que se ratifica con una evaluación media de tan sólo 3,48 puntos en la escala Likert (Likert, 1932) de 5 puntos, de esta forma, ubicando sus percepciones en la escala 3, en la mediana de la tabla. Algo similar ocurre con la quinta pregunta donde un $32,3 \%$ señala que, en las últimas dos semanas el uso de sus audífonos ha, de alguna manera, afectado su rutina diaria haciéndoles vivir ciertas dificultades auditivas, que se ratifica con una media de 3,1 puntos en la escala de Likert de 5 puntos. 


\section{CONCLUSIONES}

Desde la perspectiva del uso de audífonos, los resultados obtenidos ratifican la relevancia de las condiciones de salud previas de los adultos mayores que han recibido audífonos, puesto que, al relacionar las alteraciones sensoriales con las característica socio-demográficas de la población estudiada, se ratifica el hecho que, según las percepciones recogidas, las condiciones previas de salud parecen haber sido superadas con el uso de los audífonos al no haber tenido caídas ni presentado mareos luego de haber recibido sus audífonos.

En general, los problemas asociados a la utilización de los audífonos tienen más que ver con la adecuación de las personas a la tecnología, por los problemas de acoplamiento que requieren de dispositivos especiales para solucionarlos o por eventuales problemas de diseño, molde y ajuste auricular así como por la complejidad de las instrucciones de colocación del dispositivo en la persona.

Finalmente, las percepciones de utilidad y satisfacción recogidas en el presente estudio por el uso de audífonos por parte de loa adultos mayores, permiten confirmar lo que señala la literatura, en el sentido que, el hecho de no escuchar provoca aislamiento social y por lo tanto soledad, todo lo cual puede derivar en efectos sobre el ánimo y gatillar efectos adversos que muchas veces se traduce en depresión. Concordante con lo anterior, los adultos mayores, a pesar de las diversas dificultades que enfrentan, por los ruidos que genera el uso de sus respectivos audífonos o por sentirse diferentes respecto de sus respectivos pares, valoran positivamente los beneficios que les reporta el uso de los audífonos. Además, los resultados de este trabajo pueden ser extrapolar a ciudades de Chile con características demográficas, sociales y culturales similares a Talca. Esto es parte de un trabajo futuro.

En definitiva, los adultos mayores, valoran y aprecian en su real dimensión el haber recibido sus respectivos audífonos, reconocen la utilidad que les ha transferido a sus personas este medio de apoyo auricular y aprecian cómo les ha cambiado la vida y sus rutinas habituales, han podido volver a escuchas radio y televisión, se han reducido las caídas y reconocen estar viviendo la satisfacción de usar sus audífonos alcanzando un nivel de calidad de vida superior.

\section{REFERENCIAS}

Auditrón, Estamos para Escuchar. http://www.auditron.cl/. Acceso: 12 de Noviembre (2013).

Cañete, O., Desorden del Procesamiento Auditivo Central (DPAC), Revista de Otorrinolaringología y Cirugía de Cabeza y Cuello, 66(3), 263-273 (2006).

Castellón, A. y Sanchez del Pino, M. A., Calidad de Vida y Salud, Revista MULT Gerontología, 11(1), 12-9 (2001).

Censo Chile, Chile ING. http://www.ine.cl/cd2002/. Acceso: 11 de Noviembre (2013).

Contreras, R., Hipoacusia en el Paciente Anciano, Hospital de Especialidades Centro médico Nacional Siglo XXI, Pan-American Family Medicine Clinics, Vol. 1, 48-52 (2006).

Cox, R., Stephens, D. y Kramer, S., Translations of the International Outcome

Inventory for Hearing Aids (IOI-HA), International Journal of Audiology, 41, 3-26 (2002).

Diener, E., Subjective well-Being. Psychological Bulletin, 95, 542-575 (1984).

Diener, E., Emmons, R. A., Larsen, R. J. y Griffin, S., The Satisfaction with Life Scale, Journal of Personality Assessment, 49, 71-75 (1985).

Diener, E., Suh, E., Lucas, R. y Smith, H., Subjetive well-Being: Three Decades of Progress, Psychological Bulletin, 125 (2), 276-302 (1999).

Diener, E. y Lucas R., Explaining Differences in Societal Levels of Happiness: Relative Standards, Need Fulfillment, Culture, and Evaluation Theory, Journal of Happiness Studies: An Interdisciplinary Periodical on Subjective Well-Being, 1, 41-78 (2000).

Ferré Rey, J., Morelló-Castro, G. y Curto, B., Factores de Riesgo Involucrados en la Presbiacusia, Acta de Otorrinología Española, 53(8), 572-577 (2002). 
García, E., Flores, J. y Rodríguez, G., Análisis Factorial, Editorial La Muralla, (2000).

Gómez Pérez, G., Reflexiones en Torno al Concepto 'Salud', Procesos Psicológicos y Sociales, 2(1), (2006).

Likert, R., A Technique for the Measurement of Attitudes, Archives of Psychology, 140,1-55 (1932).

Minsal, Guía Clínica Hipoacusia Bilateral en Personas de 65 años y más que Requieren Uso de Audífono. http://www.redsalud.gov.cl/archivos/guiasges/hipoacusia.pdf. Acceso: 11 de Noviembre (2013).

Puente, G. A., Presbiacusia en: Medicina de la Comunicación Humana, Instituto de la Comunicación Humana, México, D.F., 503-516 (1994).

Ryff, C., Happiness is Everything, or Is It?, Exploration on the Meaning of Psychological well-Being, Journal of Personality and Social Psychology, 57, 1069-1081 (1989).

Ryff, C., In the Eye of the Beholder: Views of Psychological well-Being in Middle and old-Aged Adults, Psychological and Aging. 4: $195-210$ (1989).

S.E.G-G Geriatría XXI, Análisis de Necesidades y Recursos en la Atención a las Personas Mayores en España, EdIMSA, Madrid (2000).

Salas, N., Aprendiendo a Envejecer, El adulto mayor y las Problemáticas Derivadas. http://www.gerontologia.uchile.cl/docs/emma_2.htm. Acceso: 11 de Noviembre (2013).

Tamblay, N., Villalobos, I., Pastene A. y Rahal, M., Impacto Social del Uso de Audífonos en Adultos Mayores, Revista de Otorrinología y Cirugía de Cabeza y Cuello, 68(1), 21-26, Santiago, Chile, Abril (2008). 